\title{
S-Nitrosoglutathione Administration Ameliorates Cauda Equina Compression Injury in Rats
}

\author{
Anandakumar Shunmugavel ${ }^{1}$, Mushfiquddin Khan ${ }^{1}$, Marcus M. Martin², Anne G. Copay ${ }^{2}$, \\ Brian R. Subach ${ }^{2}$, Thomas C. Schuler ${ }^{2}$, Inderjit Singh ${ }^{1^{*}}$ \\ ${ }^{1}$ Department of Pediatrics Medical University of South Carolina, Charleston, USA; ${ }^{2}$ The Spinal Research Foundation, The Virginia \\ Spine Institute, Reston, USA. \\ Email: ${ }^{*}$ singhi@musc.edu
}

Received May $4^{\text {th }}, 2012$; revised June $19^{\text {th }}, 2012$; accepted June $11^{\text {th }}, 2012$

\begin{abstract}
Lumbar spinal stenosis (LSS) causes ischemia, inflammation, demyelination and results in cauda equina (CE) syndrome, with pain and locomotor functional deficits. We investigated whether exogenous administration of S-nitrosoglutathione (GSNO), an endogenous redox modulating anti-neuroinflammatory agent, hastens functional recovery in a CE compression (CEC) rat model. CEC was induced in adult female rats by the surgical implantation of two silicone blocks within the epidural spaces of L4-L6 vertebrae. GSNO ( $50 \mu \mathrm{g} / \mathrm{kg}$ body weight) was administered by gavage $1 \mathrm{~h}$ after the injury, and the treatment was continued daily thereafter. GSNO induced change in the pain threshold was evaluated for four days after the compression. Tissue analyses and locomotor function evaluation were carried out at two weeks and four weeks after the CEC respectively. GSNO significantly improved motor function in CEC rats as evidenced by an increased latency on rotarod compared with vehicle-treated CEC rats. CEC induced hyperalgesia was decreased by GSNO. GSNO also increased the expression of VEGF, reduced cellular infiltration (H\&E staining) and apoptotic cell death (TUNEL assay), and hampered demyelination (LFB staining and g-ratio). These data demonstrate that administration of GSNO after CEC decreased inflammation, hyperalgesia and cell death leading to improved locomotor function of CEC rats. The therapeutic potential of GSNO observed in the present study with CEC rats suggests that GSNO is a candidate drug to test in LSS patients.
\end{abstract}

Keywords: LSS; VEGF; Demyelination; g-Ratio; Neuroprotection

\section{Introduction}

Lumbar spinal stenosis (LSS) is a stable disorder with mild to severe disability and neurological deficits. LSS is one of the most frequent reasons for spinal surgery associated with chronic low back pain in the elderly population. In the US, 90 out of 100,000 persons older than 60 years undergo LSS related spinal surgery every year [1]. The socioeconomic burden due to LSS is huge and has been increasing rapidly, despite considerable technological advances in diagnosis [2,3]. LSS compresses cauda equina (CE) fibers over time and results in intermittent claudication/pseudoclaudication [4]. Further, CEC results in neurodegeneration and neuronal dysfunction through Wallerian-degeneration [5,6]. LSS-induced CEC interrupts normal spinal microvascular circulation $[7,8]$, and leads to secondary tissue damage from hypoxia/ischemia injury. Secondary damage also augments inflammation-mediated neuronal damage to the $\mathrm{CE}$ fibers and spinal cord. Increased synthesis of inflamma-

"Corresponding author. tory cytokines, chemokines, and matrix metalloproteinases (MMPs) during CEC are reported to result in defects in motor functions $[9,10]$. The enhanced inflammatory cytokine synthesis also hampers the recovery after CEC. Therefore, dampening the inflammatory response following LSS/CEC by pharmacological strategies will be a key approach in LSS treatment.

Decompressive laminectomy has been the most sought surgical treatment option for LSS [11]. However, the success rate of surgery is highly variable (between $45 \%$ to $72 \%$ ) [12]. Advances in therapeutic treatment of LSS are limited by the complexity of the pathology involved. In addition, there is a lack of suitable animal model to delineate the molecular mechanisms involved in the pathophysiology [13,14]. Recently, a rat animal model of CEC was developed by Takenobu et al. [15] by using silicon blocks to induce LSS. This model was further validated by Watanabe et al. [16]. In the present study, we followed the same animal model and evaluated the efficacy of GSNO therapy in treating LSS. 
GSNO, an endogenous thiol produced by the reaction of nitric oxide (NO) with glutathione (GSH), is an efficient nitrosylating agent [17]. Nitrosylation, like phosphorylation, plays a major role in several physiological and pathological processes [18,19]. Pharmacologically, GSNO protects the central nervous system (CNS) from excitotoxicity, inflammation, and reactive oxygen species (ROS) [20]. Our recent studies have proven GSNO to enhance motor function recovery and neurological function in rat animal models of traumatic brain injury (TBI) and ischemia reperfusion injury [21,22] and ameliorate spinal cord injury (SCI) induced sexual dysfunction [23]. GSNO elicited anti-inflammatory effects were attributed to the down regulation of $\mathrm{NF}-\kappa \mathrm{B}$, cell adhesion molecules, cytokines, and inducible NOS (iNOS) [21,22, 24-26]. Decreased expression of ICAM-1, ED1, and MMP-9 proteins and edema has been attributed to the enhanced integrity of blood brain barrier [21]. Furthermore, GSNO inhibits platelet activation and reduces embolization in humans [27-31]. The neuroprotective effect of GSNO has also been attributed to its ability to reduce neuronal apoptosis by inhibiting caspase- 3 activity [22,32]. GSNO's antioxidant/anti-apoptotic property is also attributed to the redox modulation [33] through increasing the level of antioxidant GSH [34] and reducing the level of oxidant peroxynitrite $[35,36]$.

In the present study, we tested the therapeutic potential of GSNO in treating CEC-induced by LSS in a rat animal model. We investigated the efficacy of GSNO therapy in locomotor functional recovery, cellular infiltration and apoptosis and expression of VEGF in the spinal cord and CE fibers of CEC rats.

\section{Methods}

\subsection{Animals and Experimental Design}

Female Sprague-Dawley rats (225 - $250 \mathrm{~g})$, purchased from Harlan Laboratories (Durham, NC) were housed in the animal facility at $12 \mathrm{~h}$ dark and light cycle. Ambient temperature was maintained at $25^{\circ} \mathrm{C}$. The animal procedures for the study were approved by the Institutional Animal Care and Use Committee (IACUC) of the Medical University of South Carolina. Animals were acclimatized for one week before the experiments began.

The experiment consisted of three groups: sham-operated (Sham), vehicle-treated CEC animals (Vehicle), and GSNO-treated CEC animals (GSNO). GSNO (World Precision Instruments, Inc., Sarasota, FL; $50 \mu \mathrm{g} / \mathrm{kg}$ body weight) was gavage fed to animals $1 \mathrm{~h}$ after LSS surgery and every $24 \mathrm{~h}$ thereafter till the end of the experiment. GSNO was freshly prepared in sterile water every day.

\subsection{Cauda Equina Compression}

CEC was induced following the method described by
Takenobu et al. [15]. Briefly, animals were anesthetized with a ketamine-xylazine cocktail $(80 \mathrm{mg} / \mathrm{kg}$ and 10 $\mathrm{mg} / \mathrm{kg}$ body weight respectively). After confirming the validity of anesthesia by toe pinching, the animals were depilated on the dorsal spine line, and the spine was exposed at the L3-S2 level. Two appropriate holes were made to expose the dura overlying the spinal cord of the L4 and L5 vertebrae. Two pieces of silicone rubber (4 $\mathrm{mm} \times 1 \mathrm{~mm} \times 1 \mathrm{~mm})($ Bentec Medical Inc., Woodland, CA) were placed into the L4 and L5 epidural spaces. The wound was irrigated with sterile phosphate buffered saline (PBS) solution, and the incision was closed in layers using polysorb 4. Sham animals underwent the same procedure except for the placement of silicone blocks. Animals were returned to cages, kept on a $37^{\circ} \mathrm{C}$ heating blanket overnight. The animals were closely monitored for symptoms of hind limb paralysis and urinary bladder incontinence. Animals that showed either hind limb paralysis or loss of tail sensation on pinching were excluded from the study.

\subsection{Locomotor Function Evaluation}

CEC-induced locomotor deficit was measured with rotarod. Latency on a rotarod was measured as described previously from our laboratory [21]. Animals were pretrained on an automated 4-lane rotarod unit supplied by Columbus Instruments (Columbus, OH). Total time in seconds that the animal could stay on the drum was recorded. Each animal was given three trials, and the mean was presented as the final data.

\subsection{Hyperalgesia and Nociception}

Pain threshold of experimental animals were measured using dynamic plantar aesthesiometer (DPA) and analgesy meter (AM). DPA (Ugo Basile, Italy) is an automated version of von Frey hair analysis used to measure changes in the development of mechanical allodynia resulting from neuropathic pain [37]. Animals were acclimatized to the DPA for about 15 minutes before recording. In addition, animals were acclimated to the testing area of DPA for 3 - 5 days before the actual experiment. Animals were placed individually in the testing area with a wire mesh floor. The instrument raised the filament through the wire mesh to touch the foot and progressively increased the force until it reached a maximum of $20 \mathrm{~g}$ of force. The foot withdrawal/latency was recorded using the software provided by the manufacturer. AM (Ugo Basile, Italy) was used to measure the influence of GSNO on nociception of CEC rats following the method described before [38]. Continuously increasing pressure was applied to the dorsal surface of the hind paws. The actual load (g) applied at the moment the animal withdrew its paw was recorded. Three trails were made on each paw with 5-minute intertest intervals. 


\subsection{Myelination and g-Ratio}

CE fibers from sham, vehicle, and GSNO-treated animals were extracted 7 weeks after the induction of CEC. The tissues were fixed overnight in $2 \%$ cacodylate glutaraldehyde and post-fixed in $2 \%$ osmium tetroxide for 2 h. Following rinsing in distilled water, the tissues were dehydrated in ascending grades of alcohol, infiltrated with propylene oxide and embedded at proper orientation [39]. Thin sections of 0.5 micron were cut with Teichert ultra microtome and stained with toluidine blue. Ultrathin sections of $70 \mathrm{~nm}$ were then cut and picked up on copper grids. Sections were stained with uranyl acetate and lead citrate. Ultra structural details were observed and recorded with a JEOL 1010 Transmission electron microscope. Myelination was determined based on the g-ratio, calculated following the procedure of Chomiak and $\mathrm{Hu}[40]$.

\subsection{Histology and Immunofluorescence}

Animals were sacrificed with an overdose of nembutal (150 $\mathrm{mg} / \mathrm{kg}$ body weight) and perfused transcardially with saline followed by $4 \%$ paraformaldehyde (PFA) in PBS, pH 7.4. Spinal cord and CE fibers were extracted as described [41], and post-fixed overnight in PFA. After fixation, the tissues were dehydrated in a series of alcohol and infiltrated with paraffin wax using Leica TP-1020 automatic tissue processor. Tissue blocks were sectioned $(5-8 \mu \mathrm{m})$ with a Leica HM-325 rotary microtome. Sections were rehydrated by passing through decreasing grades of ethanol $(100 \%, 95 \%, 60 \%, 70 \%$ and $30 \%$ ) and water. Sections were stained with hematoxylin and eosin (H\&E) [42] and luxol fast blue (LFB), as described previously [43]. Cellular infiltration was quantitated using NIH image J software.

For immunofluorescence studies, endogenous peroxidase was blocked with $3 \% \mathrm{H}_{2} \mathrm{O}_{2}$ after deparafinization and rehydration. To enhance immunoreactivity, sections were pretreated with $0.1 \%$ trypsin in a $10 \mathrm{mmol} / \mathrm{L}$ citrate buffer ( $\mathrm{pH}$ 6.0) at near boiling for 20 minutes. After blocking with normal goat serum, sections were incubated with the primary antibodies (1:200) overnight at $4^{\circ} \mathrm{C}$. The following primary antibodies were used: rabbit anti VEGF (1:200; abcam, San Francisco, CA) and PECAM-1 (1:200; Santa Cruz Biotech, Santa Cruz, CA). After washing with PBS (3 times, 10 min each), sections were incubated with Alexafluor conjugated secondary antibodies (1:100; Molecular probes, Invitrogen, Eugene, OR) for $1 \mathrm{~h}$ at room temperature. Sections were again rinsed with PBS, counterstained with DAPI (Invitrogen, Eugene, OR) and mounted with aqueous antifade solution. Immunofluorescence was analyzed using an epifluorescence microscope equipped with Axio-Vision 4.5 imaging software. Intensity of myelin staining and VEGF positive cells were quantitated using Image Pro plus 5.1 software [44].

\subsection{TUNEL Assay}

The terminal deoxynucleotidyl transferase-mediated biotinylated UTP nick end labeling (TUNEL) assay was performed using an Apoptag fluorescein in situ apoptosis detection kit (Serological Corp. Norcross, GA). TUNEL positive cells were counted in at least five microscopic frames of each section.

\subsection{Statistical Analysis}

Statistical analysis was performed using Graph pad prism 3.0 software. Statistical significance was determined using Students' t-test. Values were expressed as mean \pm SD of $\mathrm{n}$ determinations. $\mathrm{p}$ values less than 0.05 were considered statistically significant.

\section{Results}

\subsection{GSNO Ameliorates Locomotor Dysfunction}

Rats were pretrained on the rotarod for 5 days before the experiments began. All the animals were able to walk on rotarod for $280 \pm 8$ seconds on the day before the surgical induction of LSS. Animals in each group $(n=12)$ were tested on rotarod for 28 days following CEC. Latency on rotarod of different experimental groups of rats is given in Figure 1. On day 2 post injury, the vehicle group showed $44.3 \pm 8.5 \mathrm{sec}$ latency on rotarod. With the progression of natural healing, the walking efficiency increased a little further to $58.4 \pm 14.6 \mathrm{sec}$ on the 28 th day.

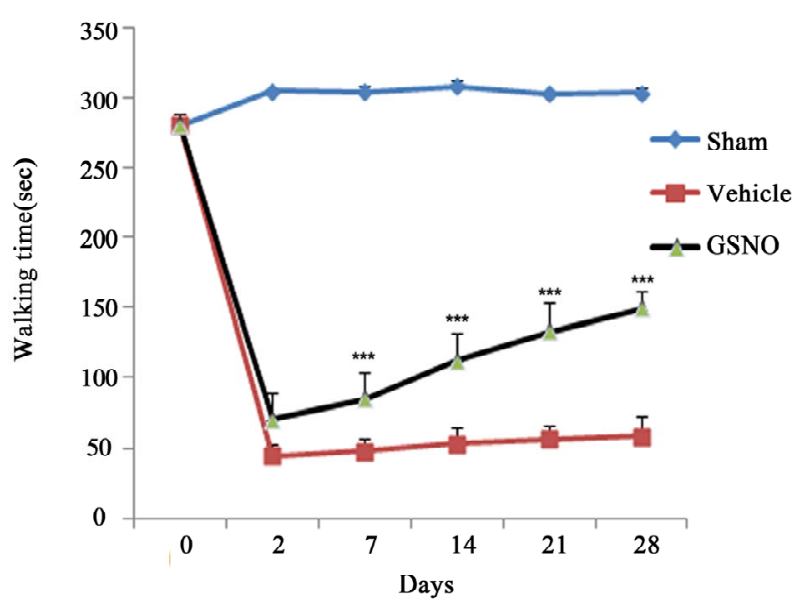

Figure 1. Effect of GSNO on CEC-mediated locomotor dysfunction in rats. GSNO treatment showed significantly improved motor function of the CEC rats when compared with vehicle group from day 7 onward. Sham rats did not show significant change in locomotor function on all the days tested. Data are expressed as mean \pm SD $(n=12$ in each group). ${ }^{* * *} \mathrm{p}<0.001$ vs. vehicle. 
The GSNO group showed significantly improved $(\mathrm{p}<$ $0.001)$ motor function starting from day $7(84.5 \pm 22.8$ seconds) to day 28 (149.7 \pm 11.6 seconds) after CEC. Sham operated animals did not show any significant change in the walking time on rotarod on any day tested (Figure 1). The data clearly show GSNO mediated amelioration of locomotor dysfunction in CEC rats.

\subsection{Gsno Decreases Demyelination and Cellular Infiltration}

Spinal cord sections proximal to the $\mathrm{CEC}$ region and $\mathrm{CE}$ fibers were processed and stained for myelin (LFB) and infiltrated cells (H\&E) on day 14 after CEC and the results are given in Figure 2. Spinal cord of the vehicle group showed increased degree of demyelination (Figure 2(a): b \& e). LFB staining in the GSNO group was comparable with that of the sham group (Figure 2(a): c, f \& a, d). GSNO also decreased cellular infiltration in the spinal tissue (Figure 2(a): i) when compared to the vehicle group (Figure 2(a): h). CEC induced decrease in LFB staining intensity and number of infiltrated cells was quantified by using Image pro plus 5.1 software and the data are given in Figures 2(b) and 2(c). Spinal cord of CEC vehicle rats showed significantly lesser intensity of LFB (b) and increased number of infiltrated cells (c).

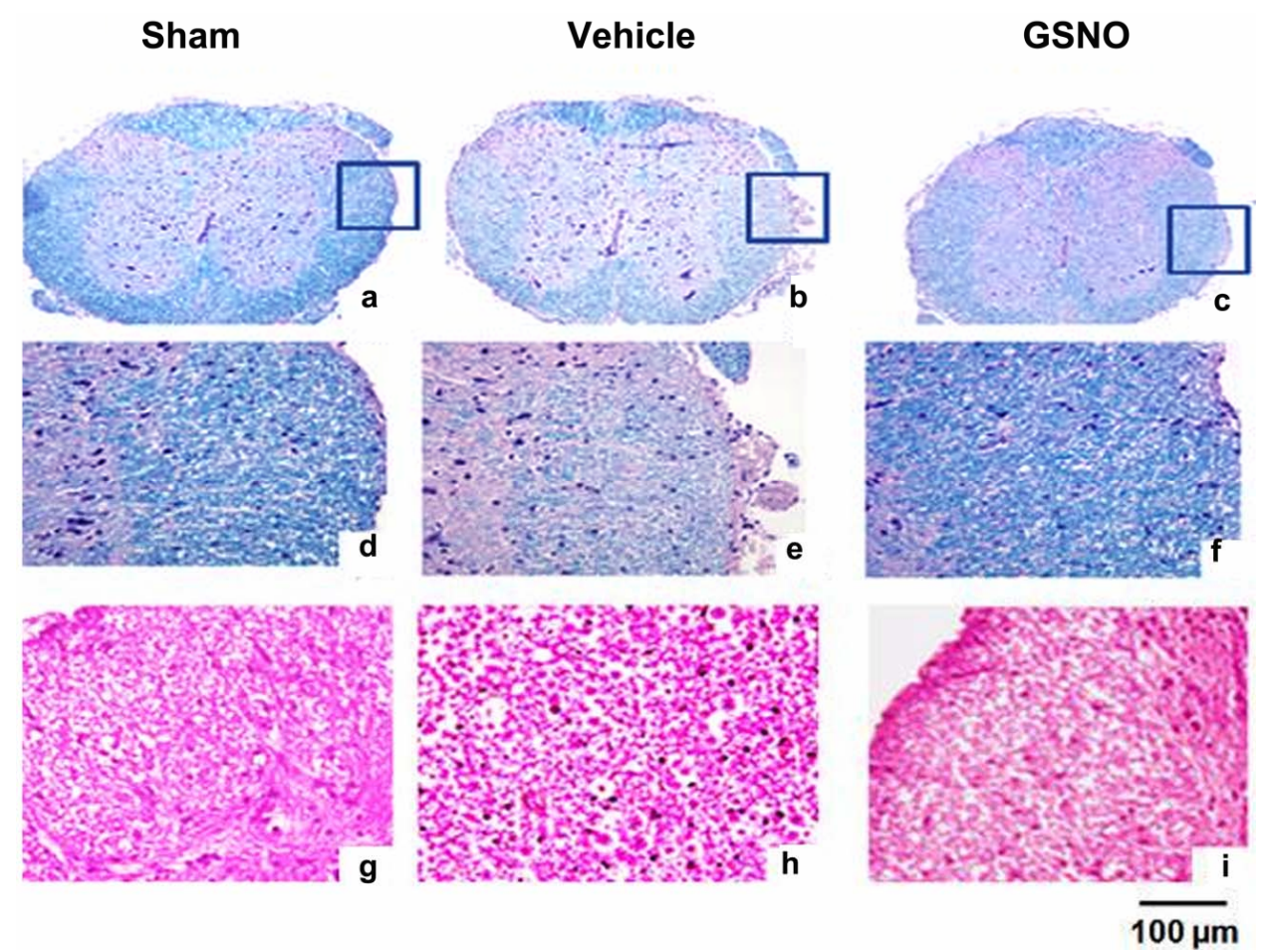

(a)

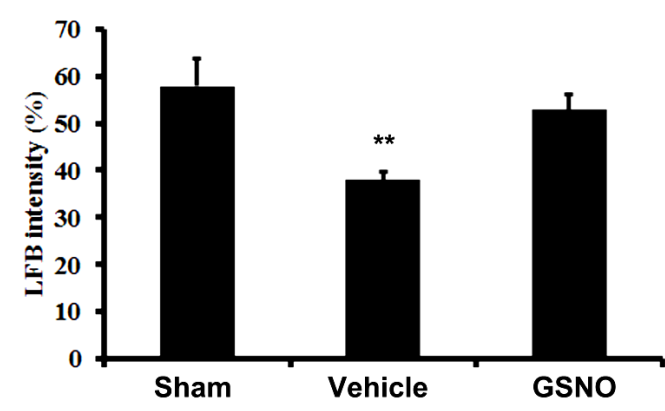

(b)

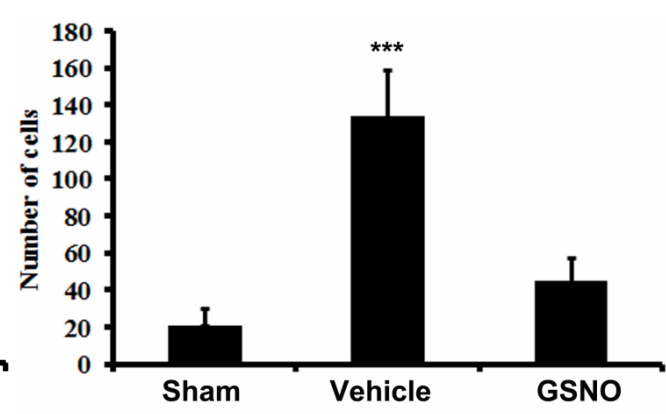

(c)

Figure 2. Effect of GSNO on CEC-induced changes in LFB histochemistry (a-f) and cellular infiltration (g-i) of spinal cord 14 days after CEC. Myelin level in the spinal cord was significantly reduced in vehicle (b \& e) compared to sham (a \& d) and GSNO rats (c \& f). Corresponding histogram on quantification of LFB staining intensity was given in Figure 2(b). Increased level of cellular infiltration was seen in spinal cord of vehicle (h) compared with sham (g) and GSNO groups (i). Photomicrographs are representative of $\mathbf{n}=\mathbf{5}$ in each group. Histogram (c) shows the quantitative difference in number of infiltrated cells in the spinal cord of different experimental groups. ${ }^{* *} p>0.01 ;{ }^{* * *} p>0.001$, vs. sham and GSNO. 
A similar trend was also seen with LFB and H\&E staining of CE fibers of different experimental groups. While the CE of vehicle group showed reduced LFB staining (Figure 3(a): b) and increased infiltration (Figure 3(a): e), the GSNO group had LFB staining (Figure 3(a): c) and cellular infiltration (Figure 3(a): f) levels similar to that of sham group (Figure 3(a): a \& d). To support further, the CEC induced demyelination and degeneration, $\mathrm{CE}$ fibers were examined with EM 7 weeks after the CEC (Figure 4). CE from sham animals had compact myelin sheath (Figure 4(a): arrow). The Schwannlike cells encircling the myelinating fiber can also be seen (Figure 4(a): arrow head). Degenerated myelin layer was seen with CE of vehicle rats (Figure 4(a): Ve- hicle). Schwann-like cells were also reduced in size, and these cells were not in close association with the neurons. Schwann-like cells showed healthy contact with the fibers also in the GSNO group, as indicated in Figure 4(a): GSNO.

As the g-ratio is a reliable factor of axonal myelination, we determined the g-ratio in the $\mathrm{CE}$ of sham, vehicle and GSNO group of rats. The g-ratio of CE fibers of sham, vehicle and GSNO rats determined in the present study were $0.67 \pm 0.06,0.71 \pm 0.05$ and $0.66 \pm 0.04$ respectively. Vehicle group has significantly higher g-ratio than the sham and GSNO groups. GSNO group showed a slightly lesser g-ratio than the sham group. However, the difference was not statistically significant (Figure 4(b)).

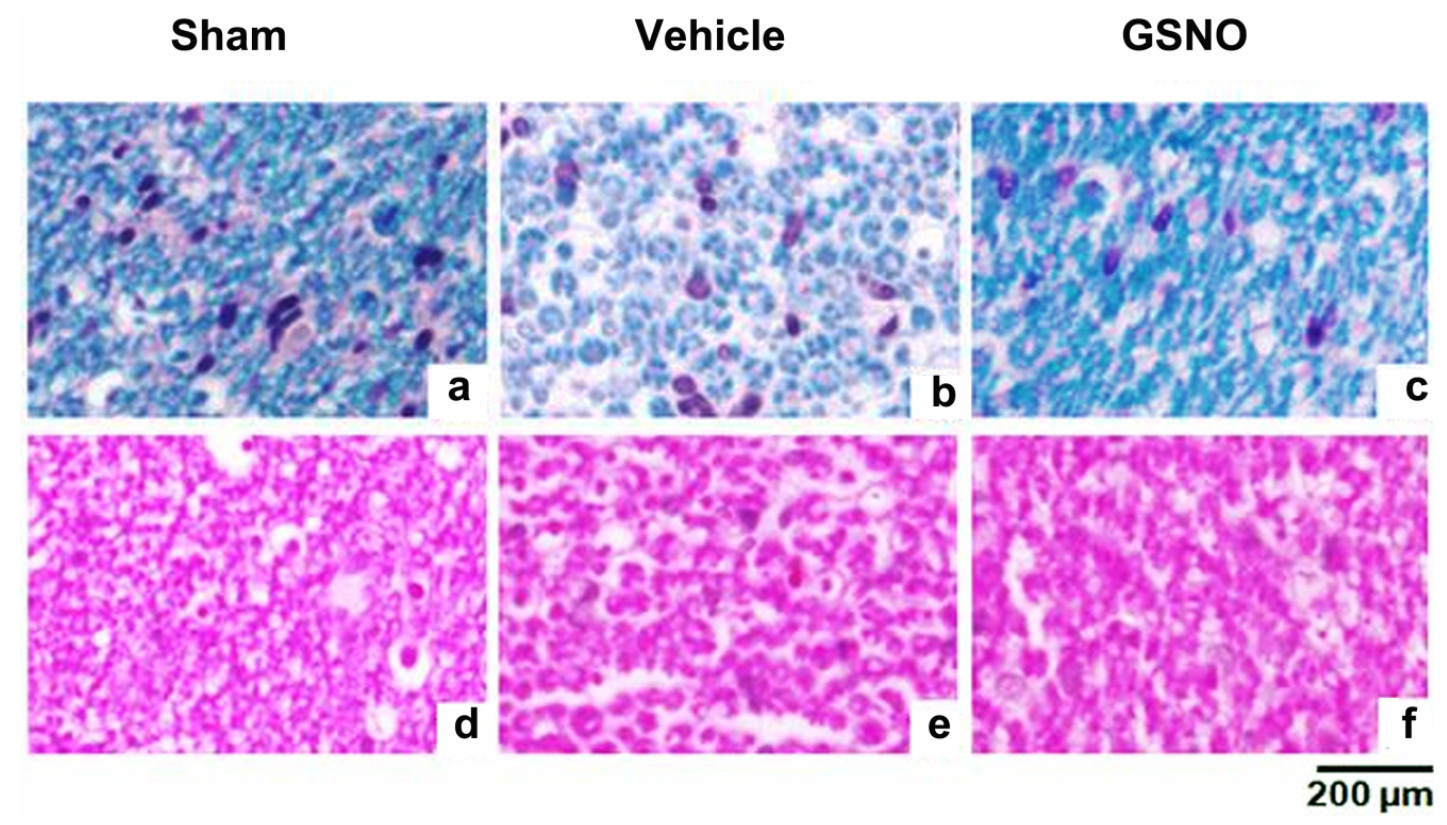

(a)

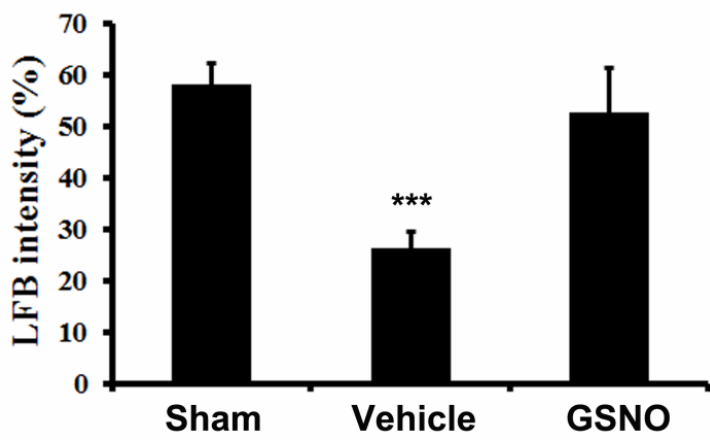

(b)

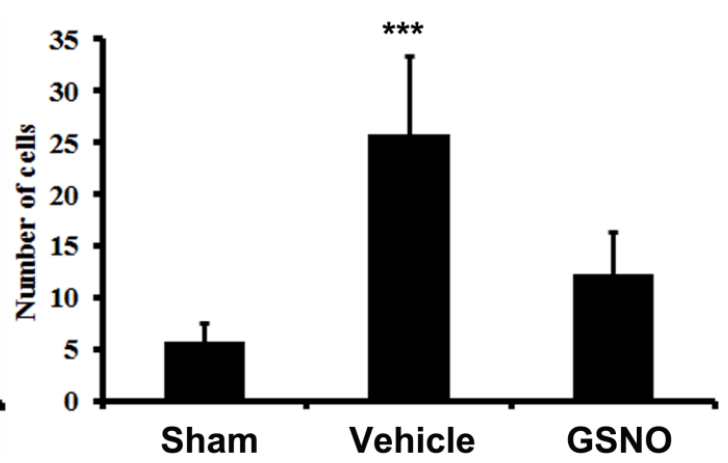

(c)

Figure 3. Effect of GSNO on CEC-induced changes in LFB histochemistry (a-c) and cellular infiltration (d-f) of CE nerve fiber 14 days after CEC. Myelin level of the nerve fiber was significantly reduced in vehicle (b) when compared with sham (a) and GSNO (c) groups. Increased cellular infiltration was also observed in vehicle (e) compared with sham (d) and GSNO (f) rats. Photomicrographs are representative of $n=5$ in each group. Histograms show the quantitative difference in LFB staining intensity and number of infiltrated cells of $\mathrm{CE}$ of different experimental groups. Vehicle group had significantly decreased level of LFB staining (b) and increased number of cellular infiltration (c)) compared with sham and GSNO animals. p $>0.001$ vs. sham and GSNO. 


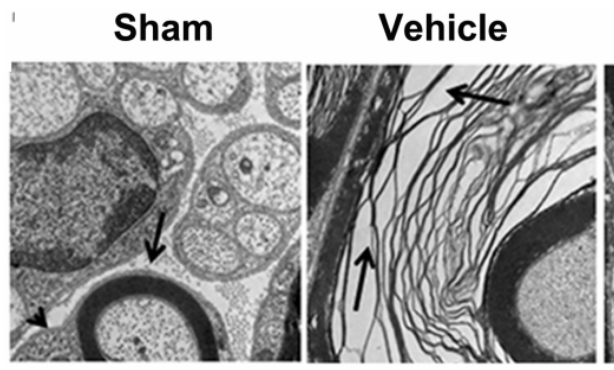

(a)

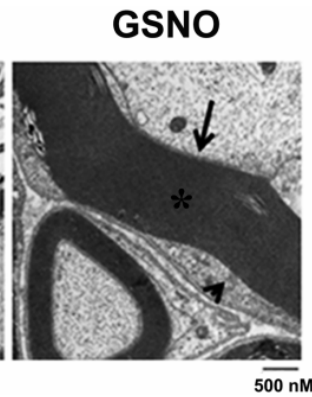

$\overline{500 \mathrm{nM}}$

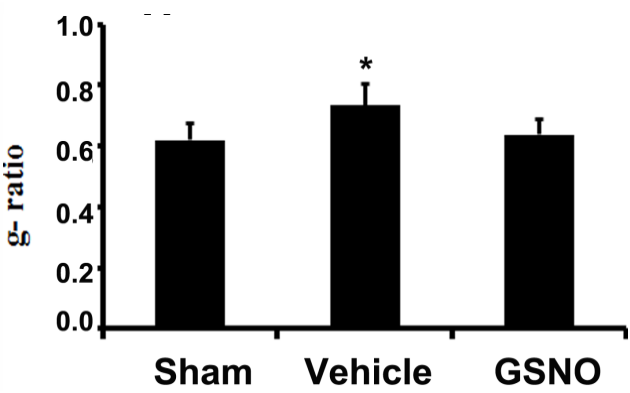

(b)

Figure 4. Effect of GSNO on CEC-induced degeneration of myelin (Figure 4(a)) and g-ratio of CE fibers (Figure 4(b)). CE fibers 7 week after CEC were processed and analyzed with EM. g-ratio was calculated with axons of $2 \mu \mathrm{m}$ thickness. CE of vehicle animals showed remarkable degeneration of myelin (a; arrow). The myelin pattern of CE fibers of GSNO animals was similar to that of sham animals. The Schwann-like cell morphology and its relationship to the nerve fiber were comparable in sham and GSNO groups (arrow head). Electron micrographs are representative of $n=4$ in each group. g-ratio of axons of vehicle was significantly higher than sham and GSNO animals (b), indicating decreased level of myelin. ${ }^{*}$ p $>0.05$ vs. sham and GSNO.

\subsection{GSNO Enhances the Pain Threshold}

Pain threshold was determined in both naïve (Figures 5(a) and (b)) and CEC (Figures 5(c) and (d)) rats. The pain threshold of naïve rats in the presence and absence of GSNO was measured at $0,1,2,4,6$ and $24 \mathrm{~h}$ using AM (Figure 5(a)) and DPA (Figure 5(b)). GSNO induced increase in the pain threshold of animals was maximum at $4-6 \mathrm{~h}$ after administration. However, $24 \mathrm{~h}$ following the treatment, the difference between the untreated and GSNO-treated animals was insignificant.

The pain threshold of sham, vehicle, and GSNO groups following CEC was measured in a way similar to naïve animals using AM (Figure 5(c)) and DPA (Figure 5(d)). Sham animals did not show any changes in pain threshold on the days measured. Vehicle rats were significantly hypersensitive to the pain stimulus. However, GSNO rats had thresholds comparable to baseline, indicating that GSNO decreases the hyperalgesia in CEC rats.

\subsection{GSNO Increases the Expression of VEGF in CE Fibers}

The expression of VEGF was determined on the compressed CE nerve fibers 14 days following CEC (Figure 6(a)). Both vehicle and GSNO groups had significantly enhanced expression of VEGF compared with the sham group. However, the GSNO group exhibited remarkably increased expression of VEGF compared with the vehicle group, as shown in Figure 6(b). VEGF expression was seen mainly in cells, also positive for PECAM-1 (Figure 6(c)) in the GSNO group, indicating that GSNO-mediated amelioration of CEC injury involves the participation of endothelial VEGF. PECAM-1 is an integral membrane glycoprotein highly expressed by endothelial cells [45].

\subsection{GSNO Reduces the Number of TUNEL Positive Cells in Spinal Cord}

Apoptotic cell death was evaluated on days 3 and 14 after CEC using a TUNEL staining assay (Figure 7(a)). TUNEL-positive cell counting was performed as previously described from our laboratory [21]. The spinal cord of vehicle rats had a significantly higher number of TUNEL-positive cells compared with the spinal cord of sham rats. The trend of increase in number of TUNELpositive cells in the vehicle was similar at day 3 and 14 after the CEC. In contrast, the spinal cord of GSNO group showed significantly reduced number of TUNELpositive cells on days 3 and day 14 after compression. The data indicate GSNO possesses an anti-apoptotic activity, as has been previously demonstrated by us in a rat model of TBI [21]. Spinal cords from the sham group did not show significant number of TUNEL-positive cells on both the days tested.

\section{Discussion}

CEC due to narrowing of the spinal canal initiates a complex series of secondary events, including inflammation, altered redox and cellular apoptosis [46-48]. Secondary injury ultimately leads to neurodegeneration and associated functional deficits $[4,16,49]$. The present study shows that GSNO treatment after the onset of LSS ameliorated motor dysfunction, reduced hyperalgesia, and inhibited tissue degeneration. Mechanistically, the protection was offered through inhibiting cellular infiltration, decreasing demyelination and apoptotic cell death, and increasing the expression of VEGF.

Locomotor deficit is the hallmark of LSS and the consequent CEC $[15,16]$. The compression causes defects in axonal myelin which affect the functioning of motor neurons [15]. Therefore, improvement of motor function 


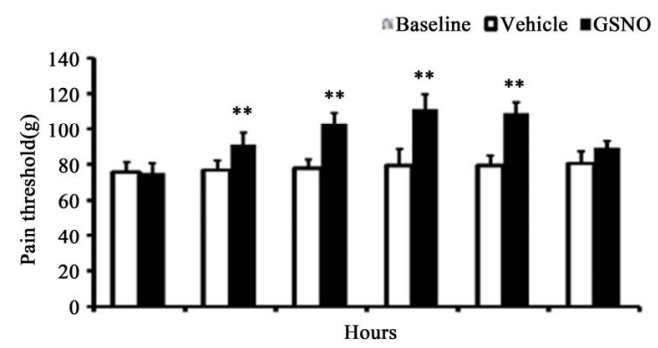

(a)

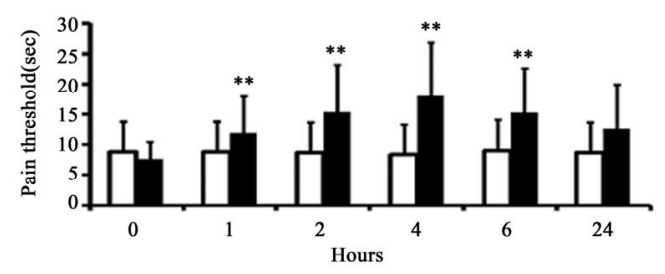

(b)

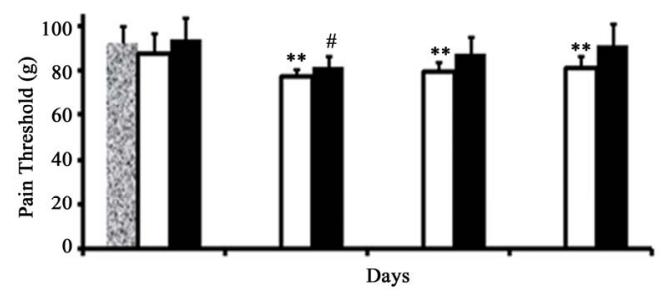

(c)

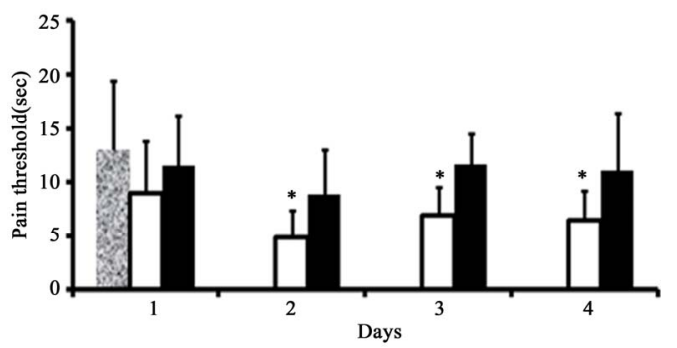

(d)

Figure 5. Effect of GSNO on pain threshold of naïve and CEC rats. Naive rats were treated either with sterile water (vehicle) or GSNO after measuring the baseline pain threshold using AM (a) as well as DPA (b). Pain threshold was measured at 1, 2, 4, 6 and 24 hours after the treatments. GSNO-mediated increase in pain threshold was noted 4 - 6 $h$ after administration. However, no significant effect was seen at $\mathbf{2 4} \mathrm{h}$. Data are presented a mean \pm SD for $\mathbf{n}=\mathbf{8}$ in each group. ${ }^{* *} p<0.01$ vs. vehicle. Effect of GSNO on pain threshold of CEC animals was given in (c) \& (d). Pain threshold was measured with AM. Base line represents the pain threshold of rats before CEC. There was a significant difference in pain threshold between the vehicle and sham groups on all days tested. However, no significant difference was observed between sham and GSNO groups from day 2. Figure 2(d) shows pain threshold of CEC rats measured with DPA. Vehicle animals showed significantly lesser pain threshold on all days tested compared with the base line. Pain threshold of GSNO animals was not significantly different from the baseline on all days tested except day 2 . Data are presented as mean \pm SD for $n=8$ in each group. ${ }^{*} p$ $<0.05,{ }^{* *} \mathrm{p}<0.005$ vs. vehicle, ${ }^{\#} \mathrm{p}<0.05$ : vs. baseline. remains the major yardstick of therapeutic interventions in humans and experimental animals after LSS. In this study, motor function deficits were observed in CEC rats from day 2 onwards (Figure 1), indicating the validity of the animal model to investigate therapeutic intervention in LSS. Deficit in motor function of CEC rats observed in the present study corroborates with the earlier report of Watanabe et al. [16]. To test the therapeutic efficacy of GSNO in LSS, CEC animals were administered with GSNO (50 $\mu \mathrm{g} / \mathrm{kg}$ body weight) 1 hour after the CEC. The dose of GSNO used in the present study was determined based on our recent studies in rat models of TBI [21] and SCI [50]. Also, we have shown that exogenous administration of GSNO $(50 \mu \mathrm{g} / \mathrm{kg})$ did not alter the other physiological parameters such as blood pressure in a rat model of TBI [51].

The g-ratio is a highly reliable factor to assess the level of axonal myelination. It has been shown previously that the g-ratio is altered during demyelinating disease [52]. The g-ratio of CE fibers of sham, vehicle and GSNO rats determined in the present study were $0.67 \pm 0.06,0.71 \pm 0.04$ and $0.66 \pm 0.04$ respectively. The g-ratio of CE fibers observed in the present study is slightly lesser than the theoretically predicted g-ratio of spinal cord (0.79) previously reported [40]. CE fibers of vehicle group showed significantly higher g-ratio, when compared to vehicle and GSNO groups. The g-ratio of GSNO group was slightly lesser than the sham group. However, the difference was not statistically significant. It has been well established that during the recovery phase of demyelinating disease axons undergo an initial hypermyelination phase and eventually revert to normal g-ratio [52]. Therefore, the present observation proves that GSNO helps axonal remyelination after spinal stenosis. However, the temporal effect of GSNO on remyelination has to be studied in detail.

Improvement of motor function in CEC rats by GSNO administration is the major finding of this study (Figure 1). A similar efficacy of GSNO in improving motor function has been reported in our previous studies on SCI and TBI animal models [21,50]; strengthening the hypothesis that GSNO enhances functional recovery in CNS injuries. Degeneration and tissue loss due to secondary injury and apoptotic cell death [49] underlie motor function deficits met with CNS diseases. We observed the loss of myelin and increased cellular infiltration in both the spinal cord (Figure 2) and CE fibers (Figure 3) of vehicle animals, indicating a profound neuronal functional deficit. Interestingly, the GSNO group had myelin levels comparable to the sham animals in both spinal cord and CE fibers (Figures 2 and 3), supporting the neuroprotective potential of GSNO therapy. Cellular infiltration was also blocked by GSNO treatment (Figures 2 and 3), supporting the anti-inflammatory property of 
GSNO. A similar effect of GSNO on myelination and cellular infiltration has been previously reported from our laboratory in TBI and SCI animal models [21,50]. Further, to support GSNO-mediated neuro-protection, CE fibers were analyzed for myelination pattern using EM study. While the myelination pattern and myelin levels were similar in sham and GSNO fibers, the vehicle fibers had significantly increased myelin degeneration and gratio (Figure 4). Furthermore, GSNO treatment reduced the number of TUNEL-positive cells in the spinal cord of CEC animals (Figure 7), supporting the neuroprotective potential of GSNO. The neuro-protective effect of GSNO has already been shown against neurotoxins [35], IR injury [22], and TBI [21]. The anti-apoptotic effect of

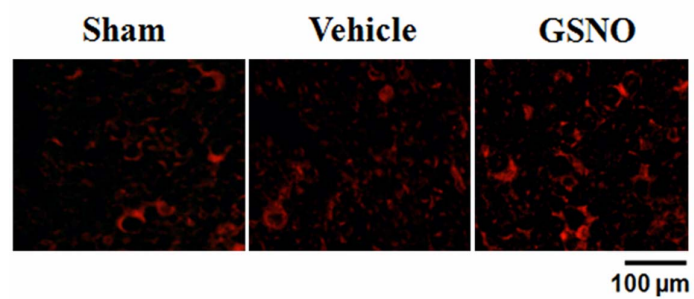

(a)

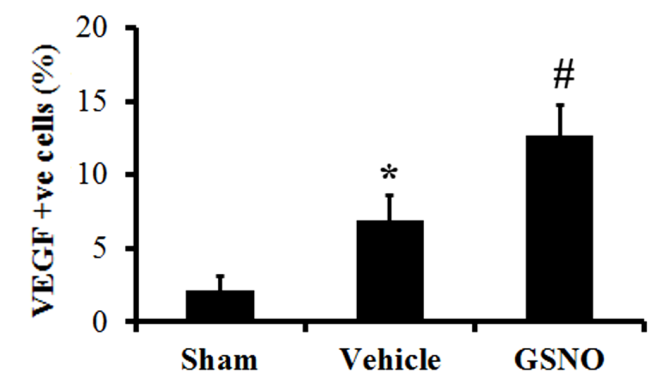

(b)

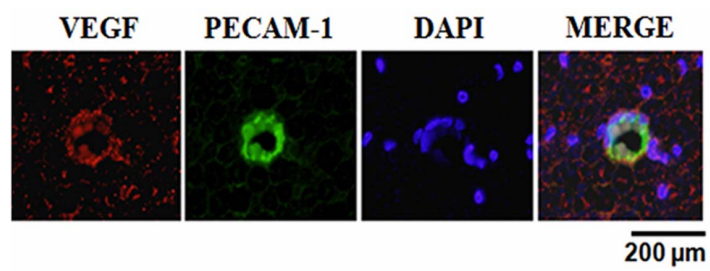

(c)

Figure 6. Effect of GSNO treatment on CEC-induced change in VEGF expression in CE fibers 14 days after $C E C$. CE fibers subjected to CEC were examined for VEGF expression by immunofluorescence staining (a). VEGF expression was elevated both in vehicle and GSNO compared with the sham rats. However, GSNO showed increased expression of VEGF compared with vehicle group. VEGF positive cells were counted in the $C E$ as explained in the material and methods section. GSNO significantly increased the VEGF expression in the CE fibers of CEC rats (b). Colocalization of VEGF with PECAM-1 (c) in GSNO group indicates that VEGF was expressed mainly in endothelial cells. Photomicrographs are representative of $\mathbf{n}=\mathbf{3}$ in each group.

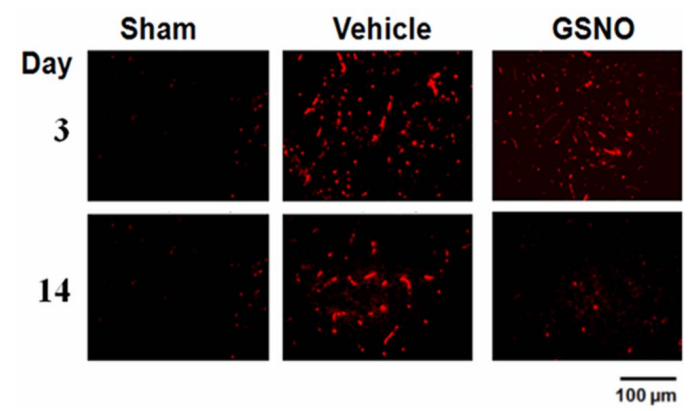

(a)

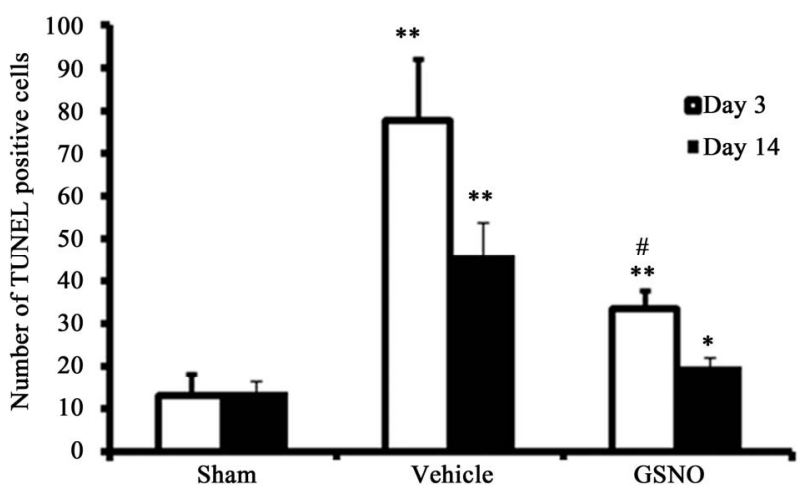

(b)

Figure 7. Effect of GSNO on CEC-induced apoptotic cell death in spinal cord. Significant increase in the number of TUNEL positive cells (determined at day 3 and 14) was observed in the spinal cord from Vehicle compared with sham group. GSNO significantly decreased the number of apoptotic cells in the spinal cord of CEC rats. Photomicrographs (a) are representative of $\mathbf{n}=\mathbf{3}$ in each group. Data of TUNEL positive cell counting (b) are expressed as mean \pm SD. ${ }^{*}$ p $<0.05,{ }^{* *}$ p $<0.01$ vs. Sham, ${ }^{\#} p<0.05$ vs. vehicle.

GSNO is shown to be regulated by blocking the activity of caspase-3 and NF- $\kappa \mathrm{B}[25,32]$. Previously, we have also reported GSNO-mediated inhibition of caspase- 3 activity in IR injury [22] and NF- $\kappa \mathrm{B}$ activity in neural and endothelial cells [22,26]. GSNO-mediated down regulation of caspase- 3 through $\mathrm{NF}-\kappa \mathrm{B}$ is invoked via S-nitrosylation [25,32]. Additionally, GSNO may stimulate the neurorepair process through the upregulation of growth and neurotropic factors including VEGF and BDNF [50,51]. In this study, the increased expression of VEGF was observed in both the GSNO and the vehicle CE fibers (Figure 7). However, the expression was remarkably high in the endothelial cells (PECAM-1 positive) of CE fibers of GSNO group compared with the vehicle group (Figure 6). Increased expression of VEGF following LSS has been reported to contribute to adaptive functional recovery [16]. Obviously, increased VEGF expression in the vehicle fibers was insufficient to achieve functional recovery in this study (Figure 6). VEGF is regulated by the transcription factor HIF $1 \alpha$, which is modulated by NO/nitrosylation $[36,53]$. There- 
fore, GSNO as an endogenous nitrosylating agent seems to be an ideal drug to activate VEGF through HIF1- $\alpha$, which in turn will stimulate the enhanced repair process of the degenerating spinal cord as well as CE fibers through angiogenesis that restores normal blood flow to the compressed ischemic spinal tissue. Because ischemia is a common occurrence following LSS [54], enhancing blood flow in the compressed area should also contribute to a speedy recovery [55-57]. Experimental drugs increasing blood flow have been shown to ameliorate LSS [58]. A study in a rat model of IR shows GSNO enhancing the cerebral blood flow and thereby improving neurological functions [22]. GSNO was reported also to increase the dermal blood flow in a rat model of diabetes [59]. Therefore, GSNO-mediated protection in LSS may occur, at least in part, through its vasculo protective effect.

Pain is a significant hallmark of pathology associated with LSS [60]. CEC elicits pain of both neurogenic and inflammatory origin. Despite a thorough understanding on the mechanisms of pain, satisfactory pain-management therapy to LSS is not yet available [60]. Moreover, the molecular mechanisms of pain involved in LSS are more complex and poorly understood $[60,61]$. In view of this, the pain threshold was evaluated in both naive and CEC rats (Figure 5) using two different modalities. GSNO treatment significantly increased the pain threshold of both naïve and vehicle rats indicating that GSNO has analgesic property in addition to neuroprotection [61]. Significant increase in tissue peroxynitrite level has been reported after spinal compression injury [46,62]. Oxidative obnoxious molecules, including peroxynitrite, are also implicated in both neurogenic and inflammatory pain. Neurogenic nociception originates due to NMDA receptor activation [60]. GSNO, via S-nitrosylation, down regulates NMDA receptors and the activity of neuronal nitric oxide synthase (nNOS) [63,64]. GSNO also reduces peroxynitrite-induced lipid peroxidation in neurodegenerative diseases $[33,35,65]$. Further, in an oxidative environment peroxynitrite is formed by nitric oxide produced from nNOS as well as iNOS [66]. Induction of iNOS and other inflammatory mediators is inhibited by GSNO via down regulation of the NF- $\kappa \mathrm{B}$ transcription factor $[22,23,26]$. The level of peroxynitrite in the brain is also decreased after GSNO treatment in rat models of IR and TBI [34,51]. All these observations strengthen the therapeutic potential of GSNO in treating neurogenic as well as inflammation-mediated pain in LSS.

In conclusion, exogenous administration of GSNO, reduces neuronal degeneration, demyelination and apoptotic cell death and also increases the pain threshold and improves motor function in a rat model of CEC. Hence, GSNO is a potential and ideal candidate to be evaluated in LSS patients.

\section{Acknowledgements}

This work was supported by grants from The Spinal Research Foundation, VA and by grants from the Betty L. Beatty and Guy E. Beatty Foundations. The work was also supported in part by grants NS-72511, NS-22576, and NS-37766 and DC00422; 07506 from the NIH, CO6 RR018823 and CO6 RR0015455 from the Extramural Research Facilities Program of the National Center for Research Resources. The authors thank Dr. Hainan Lang, Ph.D., (DC00422; 07506) Department of Pathology and Laboratory Medicine for help in histology. We are grateful to Dr. Thomas G. Smith from the MUSC Writing Center and Ms. Danielle Waulene Lowe for editing the manuscript. Ms. Joyce Bryan and Ms. Chara Williams are acknowledged for their help in animal and reagent procurement and secretarial assistance.

\section{REFERENCES}

[1] J. Steurer, A. Nydegger, U. Held, F. Brunner, J. Hodler, F. Porchet, K. Min, A. F. Mannion and B. Michel, "LumbSten: The Lumbar Spinal Stenosis Outcome Study," BMC Musculoskeletal Disorders, Vol. 11, 2010, p. 254. doi:10.1186/1471-2474-11-254

[2] S. Dagenais, J. Caro and S. Haldeman, "A Systematic Review of Low Back Pain Cost of Illness Studies in the United States and Internationally," The Spine Journal, Vol. 8, No. 1, 2008, pp. 8-20. doi:10.1016/j.spinee.2007.10.005

[3] J. N. Katz, "Lumbar Disc Disorders and Low-Back Pain: Socioeconomic Factors and Consequences," The Journal of Bone and Joint Surgery of American, Vol. 88, No. 2, 2006, pp. 21-24. doi:10.2106/JBJS.E.01273

[4] E. Siebert, H. Pruss, R. Klingebiel, V. Failli, K. M. Einhaupl and J. M. Schwab, "Lumbar Spinal Stenosis: Syndrome, Diagnostics and Treatment," Nature Reviews Neurology, Vol. 5, 2009, pp. 392-403. doi:10.1038/nrneurol.2009.90

[5] J. M. Schwab, K. Brechtel, C. A. Mueller, V. Failli, H. P. Kaps, S. K. Tuli and H. J. Schluesener, "Experimental Strategies to Promote Spinal Cord Regeneration: An Integrative Perspective," Progress in Neurobiology, Vol. 78, No. 3, 2006, pp. 91-116. doi:10.1016/j.pneurobio.2005.12.004

[6] M. E. Schwab and D. Bartholdi, "Degeneration and Regeneration of Axons in the Lesioned Spinal Cord," Physiological Reviews, Vol. 76, No. 2, 1996, pp. 319-370.

[7] O. A. Bestawros, O. H. Vreeland and M. L. Goldman, "Epidural Venography in the Diagnosis of Lumbar Spinal Stenosis," Radiology, Vol. 131, No. 2, 1979, pp. 423-426.

[8] H. N. Herkowitz, S. W. Wiesel, R. E. Booth Jr. and R. H. Rothman, "Metrizamide Myelography and Epidural Venography. Their Role in the Diagnosis of Lumbar Disc Herniation and Spinal Stenosis," Spine, Vol. 7, No. 1, 1982, pp. 55-64.

[9] J. R. Bethea, "Spinal Cord Injury-Induced Inflammation: 
A Dual-Edged Sword," Progress in Brain Research, Vol. 128, 2000, pp. 33-42. doi:10.1016/S0079-6123(00)28005-9

[10] O. N. Hausmann, "Post-Traumatic Inflammation Following Spinal Cord Injury," Spinal Cord, Vol. 41, 2003, pp. 369-378. doi:10.1038/sj.sc.3101483

[11] S. Hall, J. D. Bartleson, B. M. Onofrio, H. L. Baker Jr., H. Okazaki and J. D. O’Duffy, "Lumbar Spinal Stenosis. Clinical Features, Diagnostic Procedures, and Results of Surgical Treatment in 68 Patients," Annals of Internal Medicine, Vol. 103, No. 2, 1985, pp. 271-275.

[12] O. Niggemeyer, J. M. Strauss and K. P. Schulitz, "Comparison of Surgical Procedures for Degenerative Lumbar Spinal Stenosis: A Meta-Analysis of the Literature from 1975 to 1995," European Spine Journal, Vol. 6, No. 6, 1997, pp. 423-429. doi:10.1007/BF01834073

[13] H. Iwamoto, H. Kuwahara, H. Matsuda, A. Noriage and Y. Yamano, "Production of Chronic Compression of the Cauda Equina in Rats for Use in Studies of Lumbar Spinal Canal Stenosis," Spine, Vol. 20, 1995, pp. 2750-2757.

[14] S. Kikuchi, S. Konno, S. Kayama, K. Sato and K. Olmarker, "Increased Resistance to Acute Compression Injury in Chronically Compressed Spinal Nerve Roots. An Experimental Study," Spine, Vol. 21, 1996, pp. 25442550.

[15] Y. Takenobu, N. Katsube, M. Marsala and K. Kondo, "Model of Neuropathic Intermittent Claudication in the Rat: Methodology and Application," Journal of Neuroscience Methods, Vol. 104, No. 2, 2001, pp. 191-198. doi:10.1016/S0165-0270(00)00342-3

[16] K. Watanabe, S. Konno, M. Sekiguchi and S. Kikuchi, "Spinal Stenosis: Assessment of Motor Function, VEGF Expression and Angiogenesis in an Experimental Model in the Rat," European Spine Journal, Vol. 16, No. 11, 2007, pp. 1913-1918. doi:10.1007/s00586-007-0394-y

[17] S. P. Singh, J. S. Wishnok, M. Keshive, W. M. Deen and S. R. Tannenbaum, "The Chemistry of the S-Nitrosoglutathione/Glutathione System," Proceedings of the National Academy of Sciences of USA, Vol. 93, No. 25, 1996, pp. 14428-14433. doi:10.1073/pnas.93.25.14428

[18] M. W. Foster, T. J. McMahon and J. S. Stamler, "S-Nitrosylation in Health and Disease," Trends in Molecular and Medicine, Vol. 9, No. 4, 2003, pp. 160-168. doi:10.1016/S1471-4914(03)00028-5

[19] M. W. Foster, D. T. Hess and J. S. Stamler, "Protein S-Nitrosylation in Health and Disease: A Current Perspective," Trends in Molecular and Medicine, Vol. 15, No. 9, 2009, pp. 391-404. doi:10.1016/j.molmed.2009.06.007

[20] C. C. Chiueh, "S-Nitrosoglutathione (GSNO) Mediates Brain Response to Hypoxia," Pediatric Research, Vol. 51, 2002, p. 414. doi:10.1203/00006450-200204000-00002

[21] M. Khan, Y. B. Im, A. Shunmugavel, A. G. Gilg, R. K. Dhindsa, A. K. Singh and I. Singh, "Administration of S-Nitrosoglutathione after Traumatic Brain Injury Protects the Neurovascular Unit and Reduces Secondary Injury in a Rat Model of Controlled Cortical Impact," Journal of Neuroinflammation, Vol. 6, 2009, p. 32. doi:10.1186/1742-2094-6-32
[22] M. Khan, B. Sekhon, S. Giri, M. Jatana, A. G. Gilg, K. Ayasolla, C. Elango, A. K. Singh and I. Singh, "S-Nitrosoglutathione Reduces Inflammation and Protects Brain against Focal Cerebral Ischemia in a Rat Model of Experimental Stroke," Journal of Cerebral Blood Flow and Metabolism, Vol. 25, 2005, pp. 177-192. doi:10.1038/sj.jcbfm.9600012

[23] A. Shunmugavel, M. Khan, P. C. Chou and I. Singh, "Spinal Cord Injury Induced Arrest in Estrous Cycle of Rats Is Ameliorated by S-Nitrosoglutathione: Novel Therapeutic Agent to Treat Amenorrhea," Journal of Sexual Medicine, Vol. 9, No. 1, 2012, pp. 148-158. doi:10.1111/j.1743-6109.2011.02526.x

[24] F. D'Acquisto, M. C. Maiuri, F. de Cristofaro and R. Carnuccio, "Nitric Oxide Prevents Inducible Cyclooxygenase Expression by Inhibiting Nuclear Factor-Kappa B and Nuclear Factor-Interleukin-6 Activation," NaunynSchmiedeberg's Archives of Pharmacology, Vol. 364, No. 2, 2001, pp. 157-165. doi:10.1007/s002100100435

[25] J. D. Fortenberry, M. L. Owens, N. X. Chen and L. A. Brown, "S-Nitrosoglutathione Inhibits TNF-Alpha-Induced NFkappaB Activation in Neutrophils," Inflammation Research, Vol. 50, 2001, pp. 89-95.

[26] R. Prasad, S. Giri, N. Nath, I. Singh and A. K. Singh, "GSNO Attenuates EAE Disease by S-NitrosylationMediated Modulation of Endothelial-Monocyte Interactions," Gila, Vol. 55, No. 1, 2007, pp. 65-77. doi:10.1002/glia.20436

[27] A. S. Aledia, L. M. Tran, B. O. King, D. L. Serna, J. Eng, B. U. Jones, J. C. Chen and J. H. Roum, "S-Nitrosoglutathione Preserves Platelet Function during in Vitro Ventricular Assist Device Circulation," Asaio Journal, Vol. 48, No. 5, 2002, pp. 526-531. doi:10.1097/00002480-200209000-00015

[28] M. W. Radomski, D. D. Rees, A. Dutra and S. Moncada, "S-Nitroso-Glutathione Inhibits Platelet Activation in $\mathrm{Vi}$ tro and in Vivo," British Journal of Pharmacology, Vol. 107, No. 3, 1992, pp. 745-749. doi:10.1111/j.1476-5381.1992.tb14517.x

[29] Z. Kaposzta, A. Clifton, J. Molloy, J. F. Martin and H. S. Markus, "S-Nitrosoglutathione Reduces Asymptomatic Embolization after Carotid Angioplasty," Circulation, Vol. 106, 2002, pp. 3057-3062. doi:10.1161/01.CIR.0000041251.07332.28

[30] Z. Kaposzta, J. F. Martin and H. S. Markus, "Switching off Embolization from Symptomatic Carotid Plaque Using S-Nitrosoglutathione," Circulation, Vol. 105, 2002, pp. 1480-1484. doi:10.1161/01.CIR.0000012347.47001.97

[31] J. Molloy, J. F. Martin, P. A. Baskerville, S. C. Fraser and H. S. Markus, "S-Nitrosoglutathione Reduces the Rate of Embolization in Humans," Circulation, Vol. 98, 1998, pp. 1372-1375. doi:10.1161/01.CIR.98.14.1372

[32] S. Mohr, B. Zech, E. G. Lapetina and B. Brune, "Inhibition of Caspase-3 by S-Nitrosation and Oxidation Caused by Nitric Oxide," Biochemical Biophysical Research Communications, Vol. 238, No. 2, 1997, pp. 387-391. doi:10.1006/bbrc.1997.7304

[33] C. C. Chiueh and P. Rauhala, "The Redox Pathway of 
S-Nitrosoglutathione, Glutathione and Nitric Oxide in Cell to Neuron Communications," Free Radical Research, Vol. 31, 1999, pp. 641-650. doi:10.1080/10715769900301211

[34] M. Khan, M. Jatana, C. Elango, A. S. Paintlia, A. K. Singh and I. Singh, "Cerebrovascular Protection by Various Nitric Oxide Donors in Rats after Experimental Stroke," Nitric Oxide, Vol. 15, No. 2, 2006, pp. 114-124. doi:10.1016/j.niox.2006.01.008

[35] P. Rauhala, A. M. Lin and C. C. Chiueh, "Neuroprotection by S-Nitrosoglutathione of Brain Dopamine Neurons from Oxidative Stress," The Faseb Journal, Vol. 12, 1998, pp. $165-173$.

[36] A. Schrammel, A. C. Gorren, K. Schmidt, S. Pfeiffer and B. Mayer, "S-Nitrosation of Glutathione by Nitric Oxide, Peroxynitrite, and (*)NO/O(2)(*-)," Free Radical Biology and Medicine, Vol. 34, No. 8, 2003, pp. 1078-1088. doi:10.1016/S0891-5849(03)00038-8

[37] K. Obata, H. Yamanaka, K. Kobayashi, Y. Dai, T. Mizushima, H. Katsura, T. Fukuoka, A. Tokunaga and K. Noguchi, "Role of Mitogen-Activated Protein Kinase Activation in Injured and Intact Primary Afferent Neurons for Mechanical and Heat Hypersensitivity after Spinal Nerve Ligation," Journal of Neuroscience, Vol. 24, No. 45, 2004, pp. 10211-10222. doi:10.1523/JNEUROSCI.3388-04.2004

[38] L. O. Randall and J. J. Selitto, "A Method for Measurement of Analgesic Activity on Inflamed Tissue," Arch Int Pharmacodyn Ther, Vol. 111, No. 4, 1957, pp. 409-419.

[39] E. G. Gray, "Electron Microscopy of Presynaptic Organelles of the Spinal Cord," Journal of Anatomy, Vol. 97, 1963, pp. 101-106.

[40] T. Chomiak and B. Hu, "What Is the Optimal Value of the g-Ratio for Myelinated Fibers in the Rat CNS? A Theoretical Approach," PLoS One, Vol. 4, No. 11, 2009, Article ID: e7754. doi:10.1371/journal.pone.0007754

[41] T. Yonetake, M. Sekiguchi, S. Konno, S. Kikuchi and F. Kanaya, "Compensatory Neovascularization after Cauda Equina Compression in Rats," Spine, Vol. 33, 2008, pp. 140-145.

[42] J. Kiernan, "Interactions between Mast Cells and Nerves. Neurogenic Inflammation," Trends in Pharmacological Science, Vol. 11, No. 8, 1990, p. 316. doi:10.1016/0165-6147(90)90233-X

[43] H. Lassmann and H. M. Wisniewski, "Chronic Relapsing Experimental Allergic Encephalomyelitis: Clinicopathological Comparison with Multiple Sclerosis," Arch Neurology, Vol. 36, No. 8, 1979, pp. 490-497. doi:10.1001/archneur.1979.00500440060011

[44] S. Li, T. Li, Y. Luo, H. Yu, Y. Sun, H. Zhou, X. Liang, J. Huang and S. Tang, "Retro-Orbital Injection of FITCDextran Is an Effective and Economical Method for Observing Mouse Retinal Vessels," Molecular Vision, Vol. 17, 2011, pp. 3566-3573.

[45] F. Matsuda, H. Sakakima and Y. Yoshida, "The Effects of Early Exercise on Brain Damage and Recovery after Focal Cerebral Infarction in Rats," Acta Physiology (Oxford), Vol. 201, No. 2, 2011, pp. 275-287. doi:10.1111/j.1748-1716.2010.02174.x
[46] T. Watanabe, S. Kato, K. Sato and K. Nagata, "Nitric Oxide Regulation System in Degenerative Lumbar Disease," The Kurume Medical Journal, Vol. 52, No. 1-2, 2005, pp. 39-47. doi:10.2739/kurumemedj.52.39

[47] M. Brock and J. Ramsbacher, "Lumbar Spinal Stenosis," Critical Reviews in Neurosurgery, Vol. 8, 1998, pp. 333337. doi: $10.1007 / \mathrm{s} 003290050098$

[48] M. Sekiguchi, S. Kikuchi and R. R. Myers, "Experimental Spinal Stenosis: Relationship between Degree of Cauda Equina Compression, Neuropathology, and Pain," Spine, Vol. 29, 2004, pp. 1105-1111.

[49] J. Orendacova, D. Cizkova, J. Kafka, N. Lukacova, M. Marsala, I. Sulla, J. Marsala and N. Katsube, "Cauda Equina Syndrome," Progress in Neurobiology, Vol. 64, 2001, pp. 613-637. doi:10.1016/S0301-0082(00)00065-4

[50] P. C. Chou, A. Shunmugavel, H. E. Sayed, M. M. Desouki, S. A. Nguyen, M. Khan, I. Singh and M. Bilgen, "Preclinical Use of Longitudinal MRI for Screening the Efficacy of S-Nitrosoglutathione in Treating Spinal Cord Injury," Journal of Magnetic Resonance Imaging, Vol. 33, 2011, pp. 1301-1311. doi:10.1002/jmri.22574

[51] M. Khan, H. Sakakima, T. S. Dhammu, A. Shunmugavel, Y. B. Im, A. G. Gilg, A. K. Singh and I. Singh, "S-Nitrosoglutathione Reduces Oxidative Injury and Promotes Mechanisms of Neurorepair Following Traumatic Brain Injury in Rats," Journal of Neuroinflammation, Vol. 8, 2011, p. 78. doi:10.1186/1742-2094-8-78

[52] W. F. Blakemore, "Remyelination of the Superior Cerebellar Peduncle in the Mouse Following Demyelination Induced by Feeding Cuprizone," Journal of Neurological Science, Vol. 20, No. 1, 1973, pp. 73-83 doi:10.1016/0022-510X(73)90119-6

[53] J. Lok, P. Gupta, S. Guo, W. J. Kim, M. J. Whalen and K. van Leyen and E. H. Lo, "Cell-Cell Signaling in the Neurovascular Unit," Neurochemical Research, Vol. 32, No. 12, 2007, pp. 2032-2045. doi:10.1007/s11064-007-9342-9

[54] M. Sekiguchi, Y. Aoki, S. Konno and S. Kikuchi, "The Effects of Cilostazol on Nerve Conduction Velocity and Blood Flow: Acute and Chronic Cauda Equina Compression in a Canine Model," Spine, Vol. 33, 2008, pp. 2605 2611.

[55] S. Kobayashi, H. Baba, K. Takeno, S. Shimada, M. Kubota, T. Yayama, T. Miyazaki, K. Uchida and Y. Suzuki, "Blood Flow Analysis of Compressed Nerve Root after Intravenous Injection of Lipo-Prostaglandin E1," Journal of Orthopaedic Research, Vol. 27, 2009, pp. 1252-1257. doi:10.1002/jor.20881

[56] C. C. Chan, "Inflammation: Beneficial or Detrimental after Spinal Cord Injury?" Recent Patents on CNS Drug Discovery, Vol. 3, 2008, pp. 189-199. doi: $10.2174 / 157488908786242434$

[57] E. Holmberg, S. X. Zhang, P. D. Sarmiere, B. R. Kluge, J. T. White and S. Doolen, "Statins Decrease Chondroitin Sulfate Proteoglycan Expression and Acute Astrocyte Activation in Central Nervous System Injury," Experimental Neurology, Vol. 214, No. 1, 2008, pp. 78-86. doi:10.1016/j.expneurol.2008.07.020

[58] M. Shirasaka, B. Takayama, M. Sekiguchi, S. Konno and S. Kikuchi, "Vasodilative Effects of Prostaglandin E1 
Derivate on Arteries of Nerve Roots in a Canine Model of a Chronically Compressed Cauda Equina," BMC Musculoskeletal Disorders, Vol. 9, 2008, p. 41. doi:10.1186/1471-2474-9-41

[59] A. B. Seabra, E. Pankotai, M. Feher, A. Somlai, L. Kiss, L. Biro, C. Szabo, M. Kollai, M. G. de Oliveira and Z. Lacza, "S-Nitrosoglutathione-Containing Hydrogel Increases Dermal Blood Flow in Streptozotocin-Induced Diabetic Rats," British Journal Dermatology, Vol. 156, 2007, pp. 814-818. doi:10.1111/j.1365-2133.2006.07718.x

[60] F. T. Nickel, F. Seifert, S. Lanz and C. Maihofner, "Mechanisms of Neuropathic Pain," European Neuropsychopharmacology, Vol. 22, 2012, pp. 81-91. doi:10.1016/j.euroneuro.2011.05.005

[61] Z. Khalil, T. Liu and R. D. Helme, "Free Radicals Contribute to the Reduction in Peripheral Vascular Responses and the Maintenance of Thermal Hyperalgesia in Rats with Chronic Constriction Injury," Pain, Vol. 79, 1999, pp. 31-37. doi:10.1016/S0304-3959(98)00143-2

[62] Z. Khalil and B. Khodr, "A Role for Free Radicals and Nitric Oxide in Delayed Recovery in Aged Rats with
Chronic Constriction Nerve Injury," Free Radical Biology \& Medicine, Vol. 31, 2001, pp. 430-439. doi:10.1016/S0891-5849(01)00597-4

[63] Y. B. Choi and S. A. Lipton, "Redox Modulation of the NMDA Receptor," Cellular and Molecular Life Sciences, Vol. 57, 2000, pp. 1535-1541. doi:10.1007/PL00000638

[64] S. A. Lipton, Y. B. Choi, Z. H. Pan, S. Z. Lei, H. S. Chen, N. J. Sucher, J. Loscalzo, D. J. Singel and J. S. Stamler, "A Redox-Based Mechanism for the Neuroprotective and Neurodestructive Effects of Nitric Oxide and Related Nitroso-Compounds," Nature, Vol. 364, 1993, pp. 626-632. doi:10.1038/364626a0

[65] C. C. Chiueh, T. Andoh, A. R. Lai, E. Lai and G. Krishna, "Neuroprotective Strategies in Parkinson's Disease: Protection against Progressive Nigral Damage Induced by Free Radicals," Neurotoxicity Research, Vol. 2, 2000, pp. 293-310. doi:10.1007/BF03033799

[66] P. Pacher, J. S. Beckman and L. Liaudet, "Nitric Oxide and Peroxynitrite in Health and Disease," Physiological Reviews, Vol. 87, 2007, pp. 315-424. doi: $10.1152 /$ physrev.00029.2006 